of informed consent as 'the cutting edge of the patient autonomy movement'. He attempts to reconcile the views of its opponents and proponents by offering a clinically realistic and operational model of informed consent, in which it is treated not merely as a ritual but as a useful tool for medical management.

The first part of the book prepares the ground for the model presented in the second. It discusses the insufficiency of the legal approach to the doctrine of informed consent, analyzes the arguments of the 'paternalists' and of their critics, who defend the autonomy of patients, examines empirical studies concerning informed consent, and reflects on values that are at stake here.

The operational model provided in the second part of the book is treated as a practical application of the major theoretical work, $A$ History and Theory of Informed Consent, by R Faden and $\mathrm{T}$ Beauchamp, which also provides 'a standard by which the model can be judged' (page 72).

Wear's model comprises three stages. During the first rather complex and long stage, called Comprehensive Disclosure - which serves primarily as an insurance against a malpractice suit - the patient is provided with a lot of information (such as the diagnosis, prognosis without the treatment, recommended treatment where appropriate, identification of risks and benefits, and alternative treatments) recognizing that the patient may be unable to understand it all.

The aim of the second stage, called The Core Disclosure, 'is to present [to the patient] the essentials of the choice at hand in an approachable and palatable fashion' (page 99). So the doctor needs to focus on the basic reasons for recommending a given treatment.

Unlike the first two stages, in which the doctor may do ali the talking (if the patient chooses not to interact), during the third stage (called Assessment, Clarification, and Patient's Choice) it is the patient who is asked to speak and provide a summary of what he or she has been told. The aim of this stage is to correct and clarify the patient's actual level of understanding which will be followed by the patient's choice.

The book is written in a clear, simple and very practically oriented style. It provides dozens of helpful recommendations to practitioners regarding how to proceed in different situations (for example, when and how to talk about risks, or how to pre- sent alternative treatments), so in this respect it fulfils its promise to explain what the doctrine of informed consent amounts to at the bedside. Whether the whole model will satisfy the paternalists as well as the 'autonomists' and whether practitioners will find it attractive enough to treat it as 'a useful tool for medical management' remains to be seen.

JOANNA PASEK CSERGE, University Collete, London

\section{Medical ethics today: its practice and philosophy}

\section{BMA's Ethics, Science and}

Information Division, London, BMJ

Publishing Group, 1993, xxvii +373

pages, $£ 12.95 \mathrm{pb}$

This is the fifth in a series of handbooks on medical ethics produced by the BMA. It is an invaluable first work of reference to which everyone concerned with health care ethics should have access. Certainly every medical and nursing library should have a copy and I would recommend it to practitioners and students, as well as to those in other fields, for example, philosophy and law, who have relevant interests. It is comprehensive in scope, analytical in structure and realistic in its aims, which it pursues effectively.

While seeking to inform the reader about a wide range of issues and arguments its primary concern is practical, in effect to advise practitioners how to proceed in the face of old and new problems. That advice refers to more general moral principles but draws primarily upon law and established professional values. Significantly, the book includes as appendices the Hippocratic Oath, the International Code of Medical Ethics, the amended (1983) Declaration of Helsinki on human experimentation, and the Declaration of Tokyo on torture and punishment. A fifth appendix offers 'Useful addresses' (British Medical Association, General Medical Council, World Medical Association, etc); this would have been yet more helpful had the listing been considerably longer and included a range of centres, institutes and publications specifically concerned with medical ethics. (There is, however, a bibliography of publications by organisations and individuals; though this too could be extended, and improved by brief notes on the nature and level of at least the main works.)

Twelve broad areas are covered in as many chapters: consent and refusal; confidentiality and medical records; $\overrightarrow{\bar{A}}$ children and young people; reproduction and genetic technology; caring for the dying; cessation of treatment, nonresuscitation, aiding suicide and $\frac{\rho}{\partial}$ euthanasia; treatment and prescribing; $\stackrel{\mathbb{Q}}{\varrho}$ research; duel obligations; relations between doctors; inter-professional relations; and rationing and allocating $\stackrel{\circ}{\circ}$ resources. An additional concluding $\overrightarrow{\vec{\omega}}$ chapter examines the general issue of $\mathscr{W}$ how one arrives at answers to ethical questions in medicine and identifies $\frac{\widehat{D}}{\mathrm{D}}$ various component concerns such as professional standards and harm to $\overrightarrow{\mathrm{N}}$ others.

Each chapter is subdivided into $\overrightarrow{\mathrm{N}}$ numbered and headed sections and $\mathrm{O}$ subsections, many of the latter being less than a page in length, for exam- $\rightarrow$ ple, chapter 4 is on reproduction and genetic technology; 4.3 concerns abortion; 4.3.2.1 describes legal views $\overrightarrow{0}$ of doctors' obligations. At the end of each chapter there is a summary, usually in the form of a series of numbered points. The analytical table of contents and a detailed index make $\bar{\partial}$ it an easy matter to find things and 을 also encourage further foraging.

Clearly this is not a work to be read $\vec{F}$ progressively chapter by chapter; but $\frac{0}{3}$ equally one should not confine one's reading to individual sections. $\mathrm{A} \underset{0}{\vec{f}}$ better technique would be to start with the short concluding chapter $\overline{3}$ 'Aims and Philosophy' and then $\overparen{D}$ proceed to some particular issue, and $\frac{\sigma}{3}$ having read about that to move on to one or two related issues. Thereafter 0 one may dip in as time and need determine. Any reflective reader willo find themselves disagreeing with some points and wondering about others. That is to be expected and welcomed, and it suggests a further role for this $\mathrm{N}$ work, ie, as a source book for student $N$ seminars and discussions.

JOHN HALDANE

Department of Moral Philosophy, University of St Andrews

Disease, diagnosis and decisions

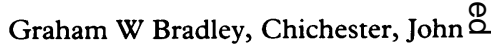
Wiley and Sons, 1993, 169 pages, $£ 14.95, \$ 23.95$

What is a disease? Bradley's primer on how to make a medical decision $\frac{\text { a }}{2}$ 